\title{
A Multidisciplinary Approach to Pediatric Liver Cancer Decreases Perioperative Complications and Improves Outcomes.
}

\author{
Richard Whitlock ${ }^{1}$, Sarah Jane Commander ${ }^{2}$, Tu-Anh Ha ${ }^{3}$, Huirong Zhu ${ }^{4}$, Jorge \\ Portuondo $^{3}$, John Goss ${ }^{3}$, Kamlesh Kukreja ${ }^{1}$, Daniel Leung ${ }^{3}$, Dolores Lopez-Terrada ${ }^{3}$, \\ Prakash Masand ${ }^{1}$, HaiThuy Nguyen ${ }^{3}$, Jed Nuchtern ${ }^{1}$, David Wesson ${ }^{1}$, Andras Heczey ${ }^{1}$, and \\ Sanjeev Vasudevan ${ }^{4}$ \\ ${ }^{1}$ Texas Children's Hospital \\ ${ }^{2}$ Duke University Medical Center \\ ${ }^{3}$ Baylor College of Medicine \\ ${ }^{4}$ Texas Children's Surgical Oncology Program, Dan L. Duncan Cancer Center, Baylor \\ College of Medicine
}

October 21, 2020

\begin{abstract}
Introduction: Partial hepatectomy in children with primary hepatic malignancies has a complication rate as high as $30 \%$. This has a negative effect on outcomes. We organized a multidisciplinary team (MDT) dedicated to the care of these patients to lower complication rates and improve outcomes. Methods: A retrospective chart review was completed for all patients $<18$ years of age who underwent liver resection at our institution between 2002 and 2019 for primary hepatic cancer. Demographic, intraoperative, postoperative, pathologic, and outcome data were analyzed for perioperative complications using the CLASSIC and ClavienDindo (CD) scales, event-free survival (EFS) and overall survival (OS). Results: Ten (13\%) of 73 patients experienced a major perioperative complication (CLASSIC or CD > 3); two (3\%) died within 30 days of surgery. After the implementation of a dedicated MDT in 2014, the major perioperative complication rate decreased from $18 \%$ to $10 \%$, and rates for all complications dropped from $52 \%$ to $20 \%(\mathrm{p}=0.005)$. Older patient age (median 62 months vs. 50 months, $\mathrm{p}=0.05)$ and the presence of pulmonary metastases at diagnosis $(\mathrm{p}=0.01)$ were associated with increased rates of major perioperative complications. Among patients with a hepatoblastoma $(\mathrm{n}=62)$, the presence of a major perioperative complication was associated with a worse EFS $(\mathrm{HR}=5.45, \mathrm{p}=0.03)$ on multivariate analysis. Patients treated following MDT implementation had improved EFS (HR=0.18, $\mathrm{p}=0.04$ ). Conclusions: Our results demonstrate that, for children with primary liver malignancies, a MDT can decrease the rate of complications of liver resection and improve the oncological outcome of hepatoblastoma.
\end{abstract}

Manuscript Word Count: 2961 Number of Tables: 6

Number of Figures: 2

Running Title: Multidisciplinary Team Liver Resection Complications

Abstract

Introduction : Partial hepatectomy in children with primary hepatic malignancies has a complication rate as high as $30 \%$. This has a negative effect on outcomes. We organized a multidisciplinary team (MDT) dedicated to the care of these patients to lower complication rates and improve outcomes.

Methods : A retrospective chart review was completed for all patients $<18$ years of age who underwent liver resection at our institution between 2002 and 2019 for primary hepatic cancer. Demographic, intra- 
operative, postoperative, pathologic, and outcome data were analyzed for perioperative complications using the CLASSIC and Clavien-Dindo (CD) scales, event-free survival (EFS) and overall survival (OS).

Results : Ten (13\%) of 73 patients experienced a major perioperative complication (CLASSIC or CD $>3$ ); two (3\%) died within 30 days of surgery. After the implementation of a dedicated MDT in 2014, the major perioperative complication rate decreased from $18 \%$ to $10 \%$, and rates for all complications dropped from $52 \%$ to $20 \%(\mathrm{p}=0.005)$. Older patient age (median 62 months vs. 50 months, $\mathrm{p}=0.05$ ) and the presence of pulmonary metastases at diagnosis $(\mathrm{p}=0.01)$ were associated with increased rates of major perioperative complications. Among patients with a hepatoblastoma $(n=62)$, the presence of a major perioperative complication was associated with a worse EFS $(\mathrm{HR}=5.45, \mathrm{p}=0.03)$ on multivariate analysis. Patients treated following MDT implementation had improved EFS (HR=0.18, $\mathrm{p}=0.04)$.

Conclusions : Our results demonstrate that, for children with primary liver malignancies, a MDT can decrease the rate of complications of liver resection and improve the oncological outcome of hepatoblastoma.

\section{Introduction}

Hepatoblastoma (HB) accounts for approximately $80 \%$ of all primary hepatic malignancies in children. ${ }^{1}$ The rest include hepatocellular carcinoma (HCC), undifferentiated embryonal sarcoma of the liver (UESL), malignant rhabdoid tumors, rhabdomyosarcoma, angiosarcoma, and germ-cell tumors. ${ }^{2}$ Surgical removal, by partial hepatectomy or total hepatectomy liver transplantation, is the mainstay of therapy for these patients. ${ }^{3}$

With a combination of chemotherapy and surgery, the survival rate of $\mathrm{HB}$ approaches $90 \%$; survival is only approximately $25 \%$ in patients with HCC, which is often chemotherapy resistant. ${ }^{4-6}$ With improved neoadjuvant chemotherapy regimens, primarily in cases of HB, partial hepatectomy is possible in about $85 \%$ of cases. ${ }^{7}$ Liver resections are technically complex procedures with perioperative complication rates exceeding $30 \%$ in larger series. ${ }^{8}{ }^{9}$ In patients with high risk HB, perioperative complications have been shown to be associated with worse overall survival. ${ }^{10}$

Even HB, the most common liver cancer in children, is rare. Only 100-125 children are diagnosed each year in North America. Because they are cared for in as many as 100 hospitals each hospital has experience with only 1-2 cases per year on average. ${ }^{11,12}$ We believe that it is critical to have a multi-disciplinary team of specialists dedicated to treating this rare cancer to ensure the highest quality of care and optimal outcomes. Implementation of such a team has the added benefit of concentrating local and regional referrals to increase center experience. Increasing center case volumes has been shown to improve outcomes of liver transplantation in adults and children. ${ }^{13,14,15}$

Because of their rarity and need for complex multi-modality therapy, we believed it was necessary in our hospital to implement a multidisciplinary treatment team (MDT) dedicated to the management of children with liver cancer. MDTs can thoroughly review each case, seek input from multiple specialties, coordinate care among the various disciplines, and create evidence-based and comprehensive treatment plans for these patients. Our children's liver cancer MDT includes dedicated pediatric oncologists, radiologists, pathologists, hepatologists, a pediatric surgeon with a special interest in hepatobiliary surgery and a pediatric liver transplant surgeonThe purpose of this study was to test the hypothesis that major perioperative complications of pediatric liver resections could be reduced with the implementation of a MDT We also sought to identify factors that increased the frequency of perioperative complications and whether perioperative complications were associated with worse short- and long-term oncologic outcomes.

\section{Methods}

\subsection{Study Design}

This was a retrospective review of all patients less than 18 years of age who underwent surgical resection of a primary liver malignancy between January 2002 and April 2019 at Texas Children's Hospital in Houston, TX. Treatments for patients prior to January 1, $2014(\mathrm{n}=33)$ were determined by individual oncology and 
surgery practitioners on a case-by-case basis. The surgeons for these cases included multiple general pediatric surgeons and transplant surgeons. After January 1, $2014(\mathrm{n}=40)$ patients were managed by a dedicated MDT that consisted of dedicated oncologists, radiologists, pathologists, hepatologists, a pediatric surgeon with a special interest in hepatobiliary surgery and a pediatric liver transplant surgeon. This team reviewed all patients with primary liver malignancies as a group and determined treatment strategies by consensus, including the decision to proceed with resection versus transplant. Throughout the study period, treatment was based on protocols of the Children's Oncology Group (COG) and the Société Internationale d'Oncologie Pédiatrique - Epithelial Liver Tumor Study Group (SIOPEL). ${ }^{16,} 17$ Patients greater than 18 years of age, and those who had benign pathology, previous liver resection or transplantation, or metastatic disease to the liver from other primary malignancies were excluded.

\subsection{Data Collection}

The study was approved by the Baylor College of Medicine Institutional Review Board. Patient records were reviewed for demographic information, age at diagnosis, intraoperative and post-operative variables including blood loss and need for transfusion, perioperative complications, post-operative hepatic function, pre- and post-operative chemotherapy regimens with time relations to surgery, histological and pathological findings, presence of preoperative metastatic disease, and patient outcomes. Also noted were types of hepatic resection performed, including non-anatomical (wedge) resection, standard hemi-hepatectomy, extended hemi-hepatectomy, or trisectionectomy. Cross-sectional imaging, including computerized tomography (CT) and magnetic resonance imaging (MRI), was used to characterize the primary tumor according to PRETEXT and POST-TEXT staging, ${ }^{18}$ assess vascular relationships, and establish the presence of metastatic disease. Intraoperative complications were graded according to a modified CLASSIC scale. ${ }^{19}$ CLASSIC grade $1 \& 2$ intraoperative complications were not collected given the highly subjective nature of identifying these complications from the operative reports of 10 surgeons. Post-operative complications were graded according to the modified Clavien-Dindo (CD) classification. ${ }^{20}$ Any complication with a CD or CLASSIC grade 3 or greater was defined as a major complication. Post-operative complications were defined as occurring within 30 days of the primary hepatic resection.

\subsection{Analysis :}

Statistical analyses were performed with SAS version 9.4 (SAS Institute, Cary, NC) and Stata version 16.0 (StataCorp, College Station, TX). Continuous variables are presented as mean \pm standard deviation and categorical variables are presented as number and percentages. Univariate comparisons were performed using the Pearson Chi-Square test, Fisher exact test, or nonparametric Wilcoxon rank-sum test, as appropriate. Overall survival rates and time to recurrence rates and groups with and without major complications were compared with the log-rank test. Only patients with a diagnosis of $\mathrm{HB}(\mathrm{n}=62)$ were included in event-free (EFS) and overall (OS) survival analysis. A p value of $<0.05$ was considered significant. Univariate analysis was performed individually for all preoperative, intraoperative, and post-operative factors associated with EFS and OS. Univariate analysis included log-rank test and univariate cox modeling. Factors that had a significant association $(\mathrm{p}<0.05)$ were selected for multivariable analysis (Tables $7 \& 8)$ via backwards selection.

\section{Results}

\subsection{Study Population and Demographic Variables}

Seventy-three pediatric patients who met the above criteria underwent surgical resection of a primary hepatic malignancy between 2002 and 2019. Thirty-three (45\%) were treated before the MDT implementation over a period of 12 years; the remaining forty (55\%) patients were seen in the 4 year and 4 month period post-MDT.

The mean age of diagnosis was 51 months (Table 1). In the pre-MDT era a total of 10 surgeons performed liver resections, an average of 3.3 cases per surgeon. In the post MDT period, 2 surgeons performed 40 liver resections. Within our patient cohort there was a male $(61 \%)$ and Hispanic (45\%) predominance. The most common malignancy encountered was HB $(n=62)$. A total of 6 patients presented with sarcoma, either 
angiosarcoma or UESL. Four patients presented with pediatric HCC; one patient had a malignant rhabdoid tumor of the liver.

\subsection{Perioperative Complications}

Seventy-one (97\%) had no intraoperative complications (Table 2a); 48 (65\%) had no post-operative complication (Table $2 \mathrm{~b})$. There were $25(34 \%)$ intraoperative and post-operative complications of any type (minor and major). Across all patients, a total of ten (14\%) major (CLASSIC/CD > 3) perioperative complications occurred. Two patients (3\%) experienced major intraoperative complications (Table 2a), one intraoperative arrhythmia and one inferior vena caval injury. A total of $8(11 \%)$ major post-operative complications occurred, which included biliary obstruction necessitating intervention, intra-abdominal fluid collection requiring drainage, small bowel obstruction, pancreatitis, bilateral pleural effusions requiring thoracentesis, cardiac arrest, prolonged post-operative ileus, and post-operative bacteremia and subsequent death (Table 3).

After the implementation of our center's MDT, the rate of major perioperative complications decreased from $18 \%$ to $10 \%$ ( $\mathrm{p}=0.31$ ) while the rate of all complications significantly decreased from $52 \%$ to $20 \%$ ( $\mathrm{p}=0.005)$ (Table 2c). The rate of major intraoperative complications decreased from $6 \%$ to $0 \%$, and the rate of major post-operative complications decreased from $15 \%$ to $10 \%$.

On univariate analysis, older patient age at diagnosis was associated with an increased likelihood of a major perioperative complication (mean $=62 \pm 41$ months) when compared to patients without major complications (mean $=50 \pm 65$ months; $\mathrm{p}=0.016$ ) The presence of pulmonary metastases at the time of diagnosis was associated with an increased incidence of major perioperative complications $(p=0.01)$. Neither PRETEXT $(\mathrm{p}=0.9)$ nor COG staging $(\mathrm{p}=0.3)$ was associated with increased complications. Although extended hemihepatectomy or trisectionectomy was associated with $32 \%(n=9)$ of all complications and $55 \%(n=5)$ of major complications, the extent of surgical resection was not shown to be associated with increased likelihood of major complications $(\mathrm{p}=0.07)$. The hospital length of stay was noted to be longer in patients who had experienced a major complication versus those who had not (median $=26$ days vs. 12 days, $\mathrm{p}=0.005$ ).

\subsection{Survival Analysis}

In order to focus on a more homogenous group of patients for survival analysis, we analyzed only HB patients $(n=62)$. Median length of follow up across all patients was 46 months. Microvascular invasion present on pathology, extent of surgical resection, and the presence of major perioperative complications were all significantly associated with worse EFS and OS on univariate analysis (Table 4). Patients with HB treated post-MDT experienced better EFS $(\mathrm{p}=0.03)$ and trended towards improved overall survival $(\mathrm{p}=0.06)$ compared to patients who were treated pre-MDT. Kaplan-Meier analysis showed a 3 year EFS of $92 \%$ for patients post-MDT compared to a $63 \% 3$ year EFS for patients pre-MDT (Figure 1). Cases with major complications had significantly worse EFS (45\%) over 3 years compared to cases with no complications $(81 \%)$ over 3 years.

These findings also held true in the multivariate analysis where MDT implementation led to significantly better $\mathrm{EFS}$ ( $\mathrm{HR}=0.18,95 \%$ C.I. $0.036-0.95, \mathrm{p}=0.02$ ). The occurrence of a major complication was independently predictive of a worse $\mathrm{EFS}(\mathrm{HR}=5.45,95 \%$ C.I. $1.1-26, \mathrm{p}=0.03)$ (Table 5$)$; neither major complications nor MDT implementation was shown to have a significant effect on OS (Table 6). Additionally, the presence of histological microvascular invasion was shown to be associated with a worse EFS \& OS, and a lack of negative surgical margins being associated with a worse OS consistent with previous studies. ${ }^{21,}{ }^{22}$

\section{Discussion}

Over the past 20 years, numerous studies have shown that higher hospital volumes are associated with lower perioperative morbidity and mortality after a number of surgical procedures. ${ }^{13-15,}{ }^{23-26}$ Several studies have recommended referral of patients to high volume centers of excellence for high-risk procedures. ${ }^{23}$ In 2015 , Rana et al. reported the findings of their survey of the Organ Procurement and Transplant Network database of over 6000 transplant candidates. They found that transplantation at a low volume center $(<5$ pediatric transplants annually) was an independent risk factor for post-transplant mortality. ${ }^{27}$ Hepatoblastoma is a 
rare malignancy with only approximately 100 cases diagnosed annually in the United States. ${ }^{28}$ There are approximately 125 pediatric cancer centers in North America; therefore, these centers see an average of only one case every other year.

Our experience suggests that an MDT can concentrate referrals, increase case volumes and improve outcomes. Prior to the implementation of our MDT, our center was seeing on average 2.8 cases per year. Ten different surgeons with varying levels of experience operated upon these patients. After the creation of our MDT we saw an average of 9.2 cases per year. Only two designated surgeons operated on these cases. Due to the increased institutional volume that followed the formation of our MDT, we have increased our familiarity with these high-risk patients, increased the volume of cases per surgeon, and improved our management of post-operative complications.

Multidisciplinary teams have been proposed as a standard model for care coordination of a variety of diseases in both children and adults. ${ }^{29-31}$ Prior work suggests MDTs also improve operative and oncologic outcomes. ${ }^{30,32,33}$ Within pediatric oncology, issues arise in the care of these medically complex patients that are often best resolved with increased communication among the treating specialties and providers. Studies have clearly shown that the MDT decision-making model is able to greatly reduce variations in treatment.$^{34}$ Additionally, studies have clearly demonstrated that MDTs reduce surgical complication rates. ${ }^{35,36}$ MDT decision-making has become standard of care at many institutions. This is reflected in the published guidelines of the American Academy of Pediatrics which highly recommend the MDT model for treatment of children with cancer. ${ }^{37}$

Prior to the implementation of a pediatric liver tumor MDT at our institution, the major perioperative complication rate for primary liver resections was $18 \%$; this decreased to $10 \%$ following implementation of our MDT and standardization of care for these patients. Although this was not statistically significant, there was a decrease in major complications requiring operative interventions and/or resulting in death. After MDT implementation, the rates of all perioperative complications combined, including minor complications, significantly decreased from $52 \%$ to $20 \%$. We hypothesize that this is due to more protocolized perioperative care of these patients from anesthesia care to post-operative intensive care unit management. As a result, the analysis revealed that the post MDT era was an independent factor associated with improved complication rates.

It has been well reported that perioperative complications can occur frequently during pediatric liver resection for primary malignancy. ${ }^{9,}$ 10, 38 Through a regression analysis of the Kid's Inpatient Database (KID) in 2014, Zwitscher et al. described that the associated major morbidity and mortality rates for pediatric hepatic resection were $20 \%$ and $5 \%$ respectively. Treatment at a high-volume center was an independent risk factor for complications that was attributed to an increased frequency of more radical resections at tertiary centers. ${ }^{9}$. Previous studies have quoted the morbidity and mortality of pediatric liver resection at $301 \%$ and $4 \%$, respectively ${ }^{38}$. Complications that are known to arise from liver resection include biliary leak, hemodynamically significant intraoperative bleeding, cholangitis, coagulopathy, and small bowel obstruction ${ }^{39}$. The overall rate of major perioperative complications in the present study was $13 \%(n=10)$. We classified major complications as any intraoperative or post-operative complication that was grade 3 or greater as referenced by either the CLASSIC (Table 2A) or CD (Table 2B) systems. The perioperative mortality rate for patients across all hepatic resections was $3 \%(\mathrm{n}=2)$ similar to that reported in the literature. ${ }^{8}, 38,40$

Older patient age and the presence of pulmonary metastatic disease at time of diagnosis were shown to be independent risk factors for major perioperative complications. Older age may be associated with larger venous, arterial, and/or biliary branches that do not effectively seal with simple cautery or sealing devices such as the harmonic scalpel or Ligasure during parenchymal transection. Additionally the livers are larger in older children; therefore, the amount of liver to transect includes a larger surface area and may increase the chance for bleeding and complications.

Studies show that roughly one fifth of HB patients present with pulmonary metastasis at the time of diagnosis, and although treatments have improved, the presence of lung metastatic disease is still one of the most 
powerful predictors of poor prognosis in HB. ${ }^{40-42}$ Pulmonary metastatic disease may serve as a surrogate marker for more advanced disease. In our study, the majority of patients who presented with lung metastases (78\%) were either PRETEXT 3 or 4 . Regardless of these associations, pulmonary metastasis was an independent predictor of complications. Additionally, it has been reported that more extensive liver resections are associated with higher incidences of post-operative complications. ${ }^{43}$ Extended hemi-hepatectomy was associated with $32 \%(\mathrm{n}=9)$ of all complications and $55 \%(\mathrm{n}=5)$ of major complications in our series, however, the extent of surgical resection was not predictive of complications $(\mathrm{p}=0.07)$. It is possible that more intensive chemotherapy or some other undescribed characteristic of these patients is leading to increased complications.

While we found that HB patients with a major perioperative complication had a worse EFS, this was not associated with worse overall survival. Our findings support other studies that demonstrated worse outcomes in HB patients undergoing resection who experienced a major complication. ${ }^{10}$ In the study by Becker et al., this association was hypothesized to have stemmed from the delay in resumption of adjuvant chemotherapy due to prolonged treatment of the perioperative complication. Within our study, there was no difference in time from surgery to resumption of chemotherapy between patients who did and did not experience major complications. We speculate that this difference was due to the expertise of our multidisciplinary team with the ability to promptly and aggressively treat complications in order to get the patient back onto adjuvant chemotherapy.

Limitations of this study include the retrospective nature, small sample size, and single-institution analysis. Due to the small sample size, it is difficult to dissect out the individual contribution of each of the changes that accompanied formation of the MDT. For example, the decreased complication rate could be primarily due to an increase in the annual case numbers per surgeon, independent of the other components of the MDT. The majority of patients treated in this study were treated after the introduction of the Children's Oncology Group study AHEP0731 $1^{44}$. As such, most patients were treated according to standardized chemotherapy protocols established by COG, however, we do accept the possibility of a cofounding variable that improved standardization of chemotherapy regimens through a MDT might have led to improved outcomes in the post MDT era. However, we do also recognize the ability to usher more patients into nationally established current treatment guidelines as a strength of the MDT model. The associations identified in this study will be further established by current ongoing international clinical trials for the treatment of pediatric liver cancer.

\section{Conclusion}

In conclusion, we believe our work brings to light many important predictive factors of perioperative complications and outcomes for children treated with partial hepatectomy for primary liver tumors. First, when classified in a systematic manner, both major and all perioperative complication rates were improved with the introduction of the MDT. Additionally, older age and presence of pulmonary metastasis were independently predictors of major perioperative complications. Lastly, MDTs appear to improve event free survival in HB patients. Given the very positive effects of MDT implementation in our institution compared to a historical pre-MDT cohort, we feel strongly that MDTs should be standard of care for the treatment of children with hepatic malignancies. This dedicated team will result in more centralization of care for these patients resulting in higher case volumes per surgeon, which will result in improved perioperative complication rates and overall outcomes.

\section{Conflicts of Interest: None}

Grant funding: This work was supported by the Macy Easom Cancer Research Foundation (S.A.V.).

Acknowledgements : We would like to thank Miriam King, M.Ed. of the Baylor College of Medicine Office of Surgical Research for providing editing support for this manuscript.

\section{References:}


1. Khan AS, Brecklin B, Vachharajani N, et al. Liver Transplantation for Malignant Primary Pediatric Hepatic Tumors. J Am Coll Surg 2017; 225(1):103-113.

2. Fernandez-Pineda I, Cabello-Laureano R. Differential diagnosis and management of liver tumors in infants. World J Hepatol 2014; 6(7):486-95.

3. Kirnap M, Ayvazoglu Soy E, Ozcay F, et al. Pediatric Liver Transplant For Hepatoblastoma: A SingleCenter Experience. Exp Clin Transplant 2017; 15(Suppl 1):50-52.

4. Khanna R, Verma SK. Pediatric hepatocellular carcinoma. World J Gastroenterol 2018; 24(35):3980-3999.

5. Weeda VB, Murawski M, McCabe AJ, et al. Fibrolamellar variant of hepatocellular carcinoma does not have a better survival than conventional hepatocellular carcinoma-results and treatment recommendations from the Childhood Liver Tumour Strategy Group (SIOPEL) experience. Eur J Cancer 2013; 49(12):2698704 .

6. Czauderna P, Garnier H. Hepatoblastoma: current understanding, recent advances, and controversies. F1000Res 2018; 7:53.

7. Agarwala S. Primary malignant liver tumors in children. Indian J Pediatr 2012; 79(6):793-800.

8. Towu E, Kiely E, Pierro A, et al. Outcome and complications after resection of hepatoblastoma. J Pediatr Surg 2004; 39(2):199-202; discussion 199-202.

9. Zwintscher NP, Azarow KS, Horton JD. Morbidity and mortality associated with liver resections for primary malignancies in children.Pediatr Surg Int 2014; 30(5):493-7.

10. Becker K, Furch C, Schmid I, et al. Impact of postoperative complications on overall survival of patients with hepatoblastoma.Pediatr Blood Cancer 2015; 62(1):24-8.

11. Trobaugh-Lotrario AD, Katzenstein HM. Chemotherapeutic approaches for newly diagnosed hepatoblastoma: past, present, and future strategies. Pediatr Blood Cancer 2012; 59(5):809-12.

12. Weldon CB, Madenci AL, Tiao GM, et al. Evaluation of the diagnostic biopsy approach for children with hepatoblastoma: A report from the children's oncology group AHEP 0731 liver tumor committee. $J$ Pediatr Surg 2020; 55(4):655-659.

13. Begg CB, Cramer LD, Hoskins WJ, et al. Impact of hospital volume on operative mortality for major cancer surgery. JAMA 1998; 280(20):1747-51.

14. Berry JG, Lieu TA, Forbes PW, et al. Hospital volumes for common pediatric specialty operations. Arch Pediatr Adolesc Med 2007; 161(1):38-43.

15. Tracy ET, Bennett KM, Danko ME, et al. Low volume is associated with worse patient outcomes for pediatric liver transplant centers. J Pediatr Surg 2010; 45(1):108-13.

16. Maibach R, Roebuck D, Brugieres L, et al. Prognostic stratification for children with hepatoblastoma: the SIOPEL experience. Eur J Cancer 2012; 48(10):1543-9.

17. Otte JB, Pritchard J, Aronson DC, et al. Liver transplantation for hepatoblastoma: results from the International Society of Pediatric Oncology (SIOP) study SIOPEL-1 and review of the world experience.Pediatr Blood Cancer 2004; 42(1):74-83.

18. Towbin AJ, Meyers RL, Woodley H, et al. 2017 PRETEXT: radiologic staging system for primary hepatic malignancies of childhood revised for the Paediatric Hepatic International Tumour Trial (PHITT). Pediatr Radiol 2018; 48(4):536-554.

19. Rosenthal R, Hoffmann H, Clavien PA, et al. Definition and Classification of Intraoperative Complications (CLASSIC): Delphi Study and Pilot Evaluation. World J Surg 2015; 39(7):1663-71. 
20. Clavien PA, Barkun J, de Oliveira ML, et al. The Clavien-Dindo classification of surgical complications: five-year experience.Ann Surg 2009; 250(2):187-96.

21. Shi Y, Commander SJ, Masand PM, et al. Vascular invasion is a prognostic indicator in hepatoblastoma. J Pediatr Surg 2017; 52(6):956-961.

22. Czauderna P, Otte JB, Aronson DC, et al. Guidelines for surgical treatment of hepatoblastoma in the modern era-recommendations from the Childhood Liver Tumour Strategy Group of the International Society of Paediatric Oncology (SIOPEL). Eur J Cancer 2005; 41(7):1031-6.

23. Ho V, Heslin MJ. Effect of hospital volume and experience on in-hospital mortality for pancreaticoduodenectomy. Ann Surg 2003; 237(4):509-14.

24. Dimick JB, Pronovost PJ, Cowan JA, et al. Surgical volume and quality of care for esophageal resection: do high-volume hospitals have fewer complications? Ann Thorac Surg 2003; 75(2):337-41.

25. Birkmeyer JD, Siewers AE, Finlayson EV, et al. Hospital volume and surgical mortality in the United States. N Engl J Med 2002; 346(15):1128-37.

26. Hannan EL, Radzyner M, Rubin D, et al. The influence of hospital and surgeon volume on in-hospital mortality for colectomy, gastrectomy, and lung lobectomy in patients with cancer. Surgery 2002; 131(1):6-15.

27. Rana A, Pallister Z, Halazun K, et al. Pediatric Liver Transplant Center Volume and the Likelihood of Transplantation. Pediatrics2015; 136(1):e99-e107.

28. Spector LG, Birch J. The epidemiology of hepatoblastoma.Pediatr Blood Cancer 2012; 59(5):776-9.

29. Brustia R, Slim K, Scatton O. Enhanced recovery after liver surgery.J Visc Surg 2019; 156(2):127-137.

30. Pillay B, Wootten AC, Crowe $\mathrm{H}$, et al. The impact of multidisciplinary team meetings on patient assessment, management and outcomes in oncology settings: A systematic review of the literature.Cancer Treat Rev 2016; 42:56-72.

31. Li ZJ, Yu JC. [Multidisciplinary Team and Nutrition Management for Bariatric Surgery]. Zhongguo Yi Xue Ke Xue Yuan Xue Bao 2018; 40(5):577-580.

32. Davison AG, Eraut CD, Haque AS, et al. Telemedicine for multidisciplinary lung cancer meetings. $J$ Telemed Telecare 2004; 10(3):140-3.

33. Junor EJ, Hole DJ, Gillis CR. Management of ovarian cancer: referral to a multidisciplinary team matters. Br J Cancer 1994; 70(2):363-70.

34. Ruhstaller T, Roe H, Thurlimann B, et al. The multidisciplinary meeting: An indispensable aid to communication between different specialities. Eur J Cancer 2006; 42(15):2459-62.

35. Carminucci AS, Ausiello JC, Page-Wilson G, et al. Outcome of Implementation of a Multidisciplinary Team Approach to the Care of Patients after Transsphenoidal Surgery. Endocr Pract 2016; 22(1):36-44.

36. Rebibo L, Marechal V, De Lameth I, et al. Compliance with a multidisciplinary team meeting's decision prior to bariatric surgery protects against major postoperative complications. Surg Obes Relat Dis 2017; 13(9):1537-1543.

37. Cantrell MA, Ruble K. Multidisciplinary care in pediatric oncology.J Multidiscip Healthc 2011; 4:171-81.

38. Grisotti G, Cowles RA. Complications in pediatric hepatobiliary surgery. Semin Pediatr Surg 2016; 25(6):388-394.

39. Weinberg L, Ianno D, Churilov L, et al. Goal directed fluid therapy for major liver resection: A multicentre randomized controlled trial.Ann Med Surg (Lond) 2019; 45:45-53. 
40. Zsiros J, Brugieres L, Brock P, et al. Dose-dense cisplatin-based chemotherapy and surgery for children with high-risk hepatoblastoma (SIOPEL-4): a prospective, single-arm, feasibility study. The Lancet Oncology 2013; 14(9):834-842.

41. Hishiki T, Watanabe K, Ida K, et al. The role of pulmonary metastasectomy for hepatoblastoma in children with metastasis at diagnosis: Results from the JPLT-2 study. J Pediatr Surg 2017; 52(12):2051-2055.

42. Meyers RL, Maibach R, Hiyama E, et al. Risk-stratified staging in paediatric hepatoblastoma: a unified analysis from the Children's Hepatic tumors International Collaboration. The Lancet Oncology2017; 18(1):122-131.

43. Shirabe K, Shimada M, Gion T, et al. Postoperative liver failure after major hepatic resection for hepatocellular carcinoma in the modern era with special reference to remnant liver volume. J Am Coll Surg 1999; 188(3):304-9.

44. Katzenstein HM, Langham MR, Malogolowkin MH, et al. Minimal adjuvant chemotherapy for children with hepatoblastoma resected at diagnosis (AHEP0731): a Children's Oncology Group, multicentre, phase 3 trial.Lancet Oncol 2019; 20(5):719-727.

Legend:

Table 1: Demographic, disease, treatment, and outcomes for all patients, patients with major peri-operative complications, and patients without major peri-operative complications

Table 2A: CLASSIC graded intraoperative complications

Table 2B: Clavien-Dindo graded post-operative complications

Table 2C Complication rates per MDT era

Table 3. Morbidity and mortality of major peri-operative complications

Table 4 Univariate analysis for event free and overall survival for hepatoblastoma patients

Table 5 Multivariate analysis for event free survival for hepatoblastoma patients

Table 6 Multivariate analysis for overall survival for hepatoblastoma patients

Figure 1: Kaplan Meyer curve for event free survival stratified by pre- and post-MDT eras for patients with hepatoblastoma

Figure 2: Kaplan Meyer curve for event free survival stratified by major complication experience for patients with hepatoblastoma

\section{Hosted file}

9-8 Table 1.pdf available at https://authorea.com/users/369131/articles/488063a-multidisciplinary-approach-to-pediatric-liver-cancer-decreases-perioperativecomplications-and-improves-outcomes

\section{Hosted file}

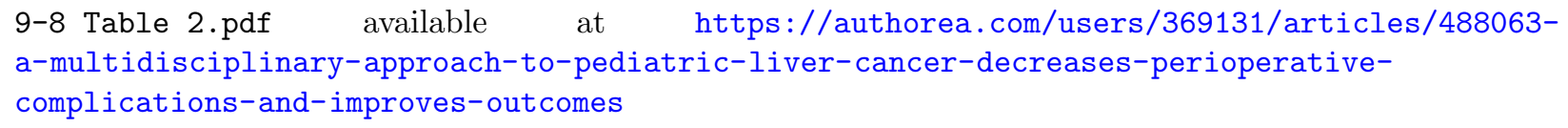

\section{Hosted file}

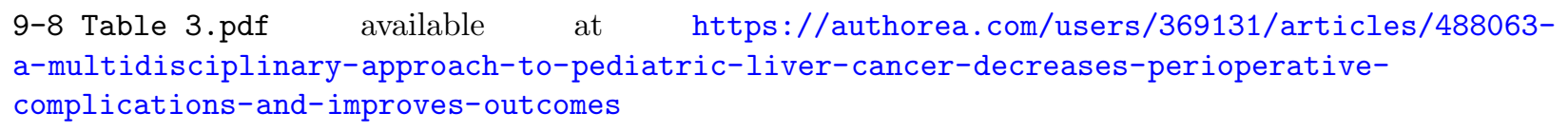




\section{Hosted file}

9-8 Table 4.pdf available at https://authorea.com/users/369131/articles/488063a-multidisciplinary-approach-to-pediatric-liver-cancer-decreases-perioperativecomplications-and-improves-outcomes

\section{Hosted file}

9-8 Table 5.pdf available at https://authorea.com/users/369131/articles/488063a-multidisciplinary-approach-to-pediatric-liver-cancer-decreases-perioperativecomplications-and-improves-outcomes

\section{Hosted file}

9-8 Table 6.pdf available at https://authorea.com/users/369131/articles/488063a-multidisciplinary-approach-to-pediatric-liver-cancer-decreases-perioperativecomplications-and-improves-outcomes 